
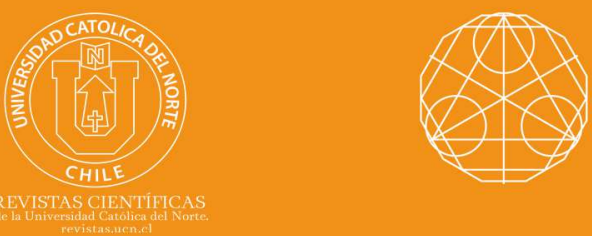

\title{
Diagonal entries of the combined matrix of sign regular matrices of order three
}

\author{
Maria T. Gassó ${ }^{1}$ (D) orcid.org/0000-0002-0471-4946 \\ Iván $\mathrm{Gil}^{2}$ \\ Isabel Giménez ${ }^{3}$ () orcid.org/0000-0003-2564-5350 \\ Máximo Santana ${ }^{4}$ (1) orcid.org/0000-0003-0611-911X

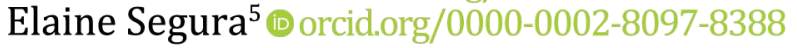

Universitat Politècnica de València, Institut de Matemàtica Multidisciplinar, Valencia, España.

1曰mgasso@imm.upv.es ; ${ }^{3}$ igimenez@imm.upv.es

Universidad Autonóma de Santo Domingo, Santo Domingo, República Dominicana.

2ఐ100082928@estuasd.edu.do ; ${ }^{4}$ msantana22@uasd.edu.do ; ${ }^{\circledR}$ esegura@uasd.edu.do

Received: Septeber 2020 | Accepted: November 2020

\section{Abstract:}

The study of the diagonal entries of the combined matrix of a nonsingular matrix $A$ has been considered by different authors for the classes of $M$ matrices, positive definite matrices and totally positive (negative) matrices. This problem appears to be difficult as the results have been done only for matrices of order three. In this work, we continue to give the characterization of the diagonal entries of the combined matrix of the remainder sign regular matrices. Thus, the problem is closed for all possible sign regular matrices of order three.

Keywords: Sign regular matrices; Combined matrices; Hadamard product.

MSC (2020): 15A24,15B48, 15B99.

\section{Cite this article as (IEEE citation style):}

M. T. Gassó, I. Gil, I. Giménez, M. Santana, and E. Segura, "Diagonal entries of the combined matrix of sign regular matrices of order three", Proyecciones (Antofagasta, On line), vol. 40, no. 1, pp. 255-271, 2021, doi: 10.22199/issn.0717-62792021-01-0016

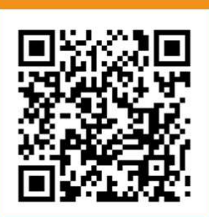

Article copyright: (C) 2021 Maria T. Gassó, Iván Gil, Isabel Giménez, Máximo Santana, and Elaine Segura. This is an open access article distributed under the terms of the Creative Commons License, which permits unrestricted use and distribution provided the original author and source are credited. 


\section{Preliminaries and notation}

The first results about the relations of the diagonal elements of a matrix $A$ and its combined matrix are given by Fiedler in [5] for $M$-matrices and in [6] for positive definite matrices. Later Fiedler and Markham in [7] give results for totally positive matrices. Bru et al. in [4] study that problem for totally negative matrices. It seems that this problem is not trivial since in all above mentioned papers the results are given for matrices of order three. In this work, we characterize which sequences of three elements can be the diagonal elements of the combined matrix of some classes of sign regular matrices. Sign regular matrices appear to be the natural generalization of totally positive or negative matrices and they have been a lot of attention by different authors (see in [9] and the references therein). Results of combined matrices of sign regular matrices are given in [3] and more recently in [1].

Throughout this paper all matrices are real. We recall that the combined matrix $\Phi(A)$ of a nonsingular matrix $A$ is defined as $\Phi(A)=A \circ A^{-T}$, where $\circ$ denotes the Hadamard product, $(A \circ B)_{i j}=a_{i j} b_{i j}$. The combined matrix $\Phi(A)=\left(c_{i j}\right)$ has the property that $\sum_{j} c_{i j}=\sum_{i} c_{i j}=1$ for each row and column. As a consequence, if $\Phi(A) \geq 0$, then $\Phi(A)$ is a doubly stochastic matrix. In $[8]$ the interesting relationship among diagonal entries and the eigenvalues of a diagonalizable matrix is given. On the other hand, applications of combined matrices to chemical problems can be viewed in [2].

A property of combined matrices that we are going to use is the following: If we multiply the matrix $A$ by a nonsingular diagonal matrix, from the left or from the right, its combined matrix $\Phi(A)$ does not change.

Given a signature $\epsilon$, that is, a real sequence $\left\{\epsilon_{1}, \ldots, \epsilon_{k}, \ldots, \epsilon_{n}\right\}$ such that $\left|\epsilon_{k}\right|=1$ for all $1 \leq k \leq n$, we recall that an $n \times n$ matrix $A$ is called sign regular with signature $\epsilon=\left(\epsilon_{k}\right)$ if for all $1 \leq k \leq n$ and $\alpha_{1}, \alpha_{2} \in Q_{k, n}$, we have

$$
\epsilon_{k} \operatorname{det}\left(A\left[\alpha_{1}, \alpha_{2}\right]\right)>0
$$

where $Q_{k, n}$ denotes the set of all increasing sequences of $k$ natural numbers less than or equal to $n$ and $A\left[\alpha_{1}, \alpha_{2}\right]$ is the submatrix of $A$ containing rows numbered by $\alpha_{1}$ and columns numered by $\alpha_{2}$ (see [9]).

\section{Results}

With matrices of order 3, we have to consider 8 possible sub-cases. However, they can be grouped into four sub-cases. This is because the combined 
matrix of the matrices $A$ and $-A$ is the same and the signatures of both matrices are opposite with the exception of the second entry because the minors of order 2 do not change.

Then we have the following equivalent pairs of signatures: (i) $(1,1,1) \leftrightarrow$ $(-1,1,-1)$, (ii) $(-1,-1,-1) \leftrightarrow(1,-1,1)$, (iii) $(1,1,-1) \leftrightarrow(-1,1,1)$ (iv) $(1,-1,-1) \leftrightarrow(-1,-1,1)$. The first case (i) was obtained by Fiedler and Markham [7] and corresponds to the totally positive matrices. The second case (ii) was studied by Bru et al.[4] when the totally negative matrices were considered.

It remains to prove the last two cases that we are going to consider in two different subsections. Thus, this problem will be closed for all sign regular matrices of order three.

\subsection{Sign regular matrices with signature $(1,1,-1)$ or $(-1,1,1)$}

We give the result for the $(1,1,-1)$ case, which is equivalent to the $(-1,1,1)$ case. To prove the main result of this case we will use the following lemma.

Lemma 1. Let $A$ be a $3 \times 3$ matrix. Then, it is $(1,1,-1)$-sign regular if and only if it is positively diagonally equivalent to the matrix

$$
S=\left[\begin{array}{rrr}
\frac{v_{1}}{v_{1}-1} & 1 & p \\
1 & 1 & 1 \\
q & 1 & \frac{v_{3}}{v_{3}-1}
\end{array}\right]
$$

where

$$
\begin{gathered}
v_{1}, v_{3}>1 \\
0<p, q<1 \\
s=1-(1-p)(1-q)\left(v_{1}-1\right)\left(v_{3}-1\right)<0
\end{gathered}
$$

Proof. $\quad(\Rightarrow)$ Suppose that the matrix

$$
A=\left[\begin{array}{lll}
a_{11} & a_{12} & a_{13} \\
a_{21} & a_{22} & a_{23} \\
a_{31} & a_{32} & a_{33}
\end{array}\right]
$$

is a $(1,1,-1)$-sign regular matrix. By the equality

$S=\left[\begin{array}{rrr}\frac{a_{11} a_{22}}{a_{12} a_{21}} & 1 & \frac{a_{13} a_{22}}{a_{12} a_{23}} \\ 1 & 1 & 1 \\ \frac{a_{22} a_{31}}{a_{21} a_{32}} & 1 & \frac{a_{22} a_{33}}{a_{23} a_{32}}\end{array}\right]=\left[\begin{array}{rrr}\frac{1}{a_{12}} & 0 & 0 \\ 0 & \frac{1}{a_{22}} & 0 \\ 0 & 0 & \frac{1}{a_{32}}\end{array}\right]\left[\begin{array}{rrr}a_{11} & a_{12} & a_{13} \\ a_{21} & a_{22} & a_{23} \\ a_{31} & a_{32} & a_{33}\end{array}\right]\left[\begin{array}{rrr}\frac{a_{22}}{a_{21}} & 0 & 0 \\ 0 & 1 & 0 \\ 0 & 0 & \frac{a_{22}}{a_{23}}\end{array}\right]$ 
we observe that the matrix $A$ is positively diagonally equivalent to the matrix $S$. Moreover, taking into account the general equivalence $\frac{x}{y}=\frac{v}{v-1} \Leftrightarrow$ $v=\frac{x}{x-y}$, we can write $S$ as follows $\mathrm{S}=\left[\begin{array}{rrr}\frac{v_{1}}{v_{1}-1} & 1 & p \\ 1 & 1 & 1 \\ q & 1 & \frac{v_{3}}{v_{3}-1}\end{array}\right]$ where $v_{i}=\frac{a_{i i} a_{22}}{a_{i i} a_{22}-a_{i 2} a_{2 i}}$, for $i=1,3, \quad p=\frac{a_{13} a_{22}}{a_{12} a_{23}}>0$ and $q=\frac{a_{22} a_{31}}{a_{21} a_{32}}>0$.

Let us see that $p, q, v_{1}$ and $v_{3}$ satisfy conditions (2.2), (2.3) and (2.4). Let $A_{i j}$ denote the $2 \times 2$ submatrix of $A$ deleting row $i$ and column $j$ from $A$. Since $A$ is $(1,1,-1)$-sign regular, we conclude the following results.

1. Since $\operatorname{det}\left(A_{33}\right)=\left(a_{11} a_{22}-a_{12} a_{21}\right)>0$, we have $v_{1}>1$.

2. From the positive sign of $\operatorname{det}\left(A_{11}\right)>0$, we obtain $v_{3}>1$.

3. Since $\operatorname{det}\left(A_{31}\right)=\left(a_{12} a_{23}-a_{13} a_{22}\right)>0$ we deduce that $p<1$. Then $0<p<1$.

4. Similarly, with $\operatorname{det}\left(A_{13}\right)=\left(a_{21} a_{32}-a_{31} a_{22}\right)>0$ we obtain that $0<q<1$.

5. By the equation (2.1) we have

$$
\begin{aligned}
& \operatorname{det}(S)=\frac{1-(1-p)(1-q)\left(v_{1}-1\right)\left(v_{3}-1\right)}{\left(v_{1}-1\right)\left(v_{3}-1\right)}<0 . \\
& \text { That is, }(1-p)(1-q)\left(v_{1}-1\right)\left(v_{3}-1\right)>1 \text { since }\left(v_{1}-1\right)\left(v_{3}-1\right)>0 .
\end{aligned}
$$

Hence, $S$ has the required form and the entries satisfy the corresponding conditions.

$(\Leftarrow)$ Conversely, suppose that $A$ is positively diagonally equivalent to matrix $S$ and the conditions (2.2), (2.3) and (2.4) are satisfied. In this case, it remains to prove that the matrix $S$ is $(1,1,-1)$-sign regular since $A$ and $S$ have the same signature. Let us verify the sign of all determinants of order 1,2 and 3 .

1. Determinants of order 1 . Note that $s_{i j}>0$ for $i, j=1,2,3$, since $p, q>0$ by $(2.3)$ and $\frac{v_{i}}{v_{i}-1}>0, i=1,3$, by $(2.2)$.

2. Determinants of order 2 .

- $\operatorname{det}\left(S_{11}\right)=\left(\frac{v_{3}}{v_{3}-1}-1\right)=\frac{1}{v_{3}-1}>0$ and $\operatorname{det}\left(S_{33}\right)=\left(\frac{v_{1}}{v_{1}-1}-1\right)>$ 0 by $(2.2)$. 
- $\operatorname{det}\left(S_{13}\right)=(1-q)>0$, and $\operatorname{det}\left(S_{31}\right)=(1-p)>0$ by $(2.3)$.

- $\operatorname{det}\left(S_{12}\right)=\left(\frac{v_{3}}{v_{3}-1}-q\right)>\frac{v_{3}}{v_{3}-1}-1=\frac{1}{v_{3}-1}>0$ by $(2.2)$.

In the same way: $\operatorname{det}\left(S_{21}\right)=\left(\frac{v_{3}}{v_{3}-1}-p\right)>0, \operatorname{det}\left(S_{32}\right)=$ $\left(\frac{v_{1}}{v_{1}-1}-p\right)>0$, and $\operatorname{det}\left(S_{23}\right)=\left(\frac{v_{1}}{v_{1}-1}-q\right)>0$.

- $\operatorname{det}\left(S_{22}\right)=\left(\frac{v_{1}}{v_{1}-1} \frac{v_{3}}{v_{3}-1}-p q\right)>0$ since $\left(\frac{v_{1}}{v_{1}-1}-p\right)>0$ and $\left(\frac{v_{3}}{v_{3}-1}-q\right)>0$ by $(2.3)$.

3. Determinant of $S$. It is clear that $\operatorname{det}(S)<0$ by $(2.2)$ and $(2.4)$.

Therefore, $S$ is sign regular with signature $(1,1,-1)$ and so the given matrix A.

Remark 1. It is well known that a necessary and sufficient condition for the system of equations $x y=f,(1-x)(1-y)=g$ has solutions $x, y \in] 0,1[$ is that $\sqrt{f}+\sqrt{g} \leq 1$.

Theorem 1. A necessary and sufficient condition for three numbers, $u_{1}, u_{2}, u_{3}$, to be the diagonal entries of the combined matrix of a $(1,1,-1)$-sign regular matrix is that $u_{i}<0, \quad i=1,2,3$, and

$$
u_{1}+u_{3}-u_{2}-1>0
$$

Proof. $\quad(\Rightarrow)$ Note that $u_{i}<0, i=1,2,3$, since the diagonal entries of the combined matrix of a $(1,1,-1)$-sign regular matrix $A$ are

$$
u_{i}=\frac{a_{i i} \operatorname{det} A_{i i}}{\operatorname{det} A}, \quad i=1,2,3 .
$$

Recall that our $(1,1,-1)$-sign regular matrix is positively diagonally equivalent to the matrix $S$ given in (2.1) with the three conditions (2.2), (2.3) and (2.4) by Lemma 1. The combined matrix of $S, \Phi(S)$ has the following diagonal entries:

$$
u_{1}=\frac{v_{1}}{s}, \quad u_{2}=\frac{v_{1} v_{3}-p q\left(v_{1}-1\right)\left(v_{3}-1\right)}{s}, \quad u_{3}=\frac{v_{3}}{s}
$$

where

$$
s=1-(1-p)(1-q)\left(v_{1}-1\right)\left(v_{3}-1\right)<0,
$$


which is the numerator of $\operatorname{det}(S)$. From conditions $(2.2)-(2.4)$ we deduce that $u_{i}<0, \quad i=1,2,3$.

Let us verify the second condition of the three numbers $u_{1}, u_{2}$ and $u_{3}$.

$$
\begin{aligned}
u_{1}+u_{3}-u_{2}-1 & =\frac{1}{s}\left(v_{1}+v_{3}-v_{1} v_{3}+p q\left(v_{1}-1\right)\left(v_{3}-1\right)-s\right) \\
& =\frac{1}{s}\left(v_{1}+v_{3}-v_{1} v_{3}+(p q+(1-p)(1-q))\left(v_{1}-1\right)\left(v_{3}-1\right)-1\right) \\
& =\frac{1}{s}\left((p(q-1)+q(p-1))\left(v_{1}-1\right)\left(v_{3}-1\right)\right)>0
\end{aligned}
$$

using conditions $(2.2)-(2.4)$.

With the given conditions for the three numbers $u_{1}, u_{2}$ and $u_{3}$ we are going to built a matrix $S$ given in Lemma 1 with the structure of (2.1) and conditions (2.2)-(2.4) such that $u_{1}, u_{2}, u_{3}$ are the diagonal entries of its combined matrix $\Phi(S)$. For that, let

$$
u_{1}=\frac{v_{1}}{s}, \quad u_{2}=\frac{v_{1} v_{3}-p q\left(v_{1}-1\right)\left(v_{3}-1\right)}{s}, \quad u_{3}=\frac{v_{3}}{s}
$$

with the unknowns $v_{1}, v_{3}, p, q$. The variable $s$ is defined in (2.7). These unknowns define the entries of matrix $S$. Observe that $u_{2} s=-p q\left(v_{1}-\right.$ 1) $\left(v_{3}-1\right)+v_{1} v_{3}$ implies

$$
p q=\frac{-u_{2} s+u_{1} u_{3} s^{2}}{\left(u_{1} s-1\right)\left(u_{3} s-1\right)} .
$$

From definition of $s$ in (2.7), it follows

$$
(1-p)(1-q)=\frac{(1-s)}{\left(u_{1} s-1\right)\left(u_{3} s-1\right)} .
$$

Then, to determine $p$ and $q$ we are going to work with (2.9) and (2.10).

Using the equivalence of the Remark 1 , the system given by equations (2.9) and (2.10) has solutions $p, q \in] 0,1[$ if and only if

$$
\sqrt{\frac{-u_{2} s+u_{1} u_{3} s^{2}}{\left(u_{1} s-1\right)\left(u_{3} s-1\right)}}+\sqrt{\frac{1-s}{\left(u_{1} s-1\right)\left(u_{3} s-1\right)}} \leq 1 .
$$

Let us see if the above inequality holds for the value $s=\frac{u_{2}}{u_{1} u_{3}}$. In this case, the equation (2.12) reduces to 


$$
\sqrt{\frac{1-s}{\left(u_{1} s-1\right)\left(u_{3} s-1\right)}} \leq 1 \Leftrightarrow 0 \leq \frac{1-s}{\left(u_{1} s-1\right)\left(u_{3} s-1\right)} \leq 1
$$

which is well defined. In fact

$$
\left(u_{1} s-1\right)\left(u_{3} s-1\right)=1-\frac{u_{2}}{u_{1}}-\frac{u_{2}}{u_{3}}+\frac{u_{2}^{2}}{u_{1} u_{3}}=\frac{u_{1} u_{3}-u_{2} u_{3}-u_{2} u_{1}+u_{2}^{2}}{u_{1} u_{3}}>0
$$

since

$$
u_{1} u_{3}-u_{2} u_{3}-u_{2} u_{1}+u_{2}^{2}=u_{1} u_{3}+u_{2}\left(-u_{3}-u_{1}+u_{2}+1\right)-u_{2}>0
$$

because $u_{i}<0$ for $i=1,2,3$ and $-1+u_{3}+u_{1}-u_{2}>0$. Note that the discriminant of $(2.12)$ is positive.

Moreover,

$$
\begin{aligned}
(1-s)-\left(u_{1} s-1\right)\left(u_{3} s-1\right) & =\left(1-\frac{u_{2}}{u_{1} u_{3}}\right)-\left(1-\frac{u_{2}}{u_{1}}-\frac{u_{2}}{u_{3}}+\frac{u_{2}^{2}}{u_{1} u_{3}}\right) \\
& =\frac{u_{2}\left(-1+u_{3}+u_{1}-u_{2}\right)}{u_{1} u_{3}}<0,
\end{aligned}
$$

since $u_{i}<0$ for $i=1,2,3$ and the condition (2.6), which means that the inequality (2.11) holds with $s=\frac{u_{2}}{u_{1} u_{3}}$. Then, our equations (2.9) and (2.10) have solutions for some $p<1$ and $0<q<1$.

In addition, $v_{1}=u_{1} s=\frac{u_{2}}{u_{3}}>1$ and $v_{3}=u_{3} s=\frac{u_{2}}{u_{1}}>1$ since the inequality (2.6) and $u_{i}<0, i=1,2,3$.

Finally, it can be computed that the diagonal elements of the combined matrix of $S$ are

$$
\operatorname{diag}(\Phi(S))=\left(\frac{v_{1}}{s}, \frac{v_{1} v_{3}-p q\left(v_{1}-1\right)\left(v_{3}-1\right)}{s}, \frac{v_{3}}{s}\right),
$$

which are precisely the elements $\left(u_{1}, u_{2}, u_{3}\right)$ of equations 3 equ. Then, we have found a solution for the unknowns $v_{1}, v_{3}, p$ and $q$ with the value $s=\frac{u_{2}}{u_{1} u_{3}}$ in which case $\operatorname{det}(S)<0$. However, $p$ or $q$ must be zero. It is easy to check that the result follows with $s=\frac{u_{2}}{u_{1} u_{3}}-\epsilon$ with the appropriate value for $\epsilon>0$, in which case $0<p<1$ and $0<q<1$. Note that with these conditions the matrix $S$ is $(1,1,-1)$-sign regular by Lemma 1 . 
Example 1. $(\Rightarrow)$ Let the $(1,1,-1)$-sign regular matrix

$$
S=\left[\begin{array}{rrr}
1.2 & 1 & 0.2 \\
1 & 1 & 1 \\
0.5 & 1 & 2
\end{array}\right]
$$

where $\left.v_{1}=6, v_{3}=2, p, q \in\right] 0,1[$ and $s=-1$. Its combined matrix is

$$
\Phi(S)=\left[\begin{array}{rrr}
-6 & 7.5 & -0.5 \\
9 & -11.5 & 3.5 \\
-2 & 5 & -2
\end{array}\right]
$$

and its diagonal entries are negative and satisfy

$$
u_{1}+u_{3}-u_{2}-1=2.5>0 .
$$

$(\Leftarrow)$ Let the three negative numbers $u_{1}=-6, u_{2}=-17$ and $u_{3}=-6$. Note that $u_{1}+u_{3}-u_{2}-1=4>0$.

According to the proof of Theorem 1, we can choose $s=\frac{u_{2}}{u_{1} u_{3}}-\epsilon=-0.5$. We obtain the parameters $p, q \in] 0,1[$ solving the system (2.9)-(2.10), and the parameters $v_{1}, v_{3}>1$ solving (2.8). So, we construct the matrix

$$
S=\left[\begin{array}{rrr}
1.5 & 1 & 0.25 \\
1 & 1 & 1 \\
0.5 & 1 & 1.5
\end{array}\right]
$$

with all conditions of Lemma 1 fulfilled. Then , $u_{1}, u_{2}, u_{3}$ are the diagonal entries of the combined matrix of the $(1,1,-1)$-sign regular matrix $S$ :

$$
\Phi(S)=\left[\begin{array}{rrr}
-6 & 8 & -1 \\
10 & -17 & 8 \\
-3 & 10 & -6
\end{array}\right]
$$

2.2. Sign regular matrices with signature $(1,-1,-1)$ or $(-1,-1,1)$

Now, we study the last case. In particular, of these two equivalent cases, we will consider the case with signature $(1,-1,-1)$. Let us start with the following result.

Lemma 2. Let $A=\left[a_{i j}\right]$ be a $3 \times 3$ matrix, where $a_{i j}>0, i, j=1,2,3$. Then, $A$ is $(1,-1,-1)$-sign regular if and only if it is positively diagonally equivalent to the matrix

$$
T=\left[\begin{array}{rrr}
\frac{v_{1}}{v_{1}-1} & 1 & p \\
1 & 1 & 1 \\
q & 1 & \frac{v_{3}}{v_{3}-1}
\end{array}\right]
$$




$$
\begin{gathered}
v_{1}, v_{3}<0 \\
p, q>1 \\
t=1-(p-1)(q-1)\left(v_{1}-1\right)\left(v_{3}-1\right)<0
\end{gathered}
$$

Proof. $\quad(\Rightarrow)$ Reasoning as in Lemma 1 we obtain a matrix $T$ positively equivalent to $A$ given by $\mathrm{T}=\left[\begin{array}{rrr}\frac{v_{1}}{v_{1}-1} & 1 & p \\ 1 & 1 & 1 \\ q & 1 & \frac{v_{3}}{v_{3}-1}\end{array}\right]$ where

$$
v_{i}=\frac{a_{i i} a_{22}}{a_{i i} a_{22}-a_{i 2} a_{2 i}} \text { for } i=1,3, \quad p=\frac{a_{13} a_{22}}{a_{12} a_{23}} \text { and } q=\frac{a_{22} a_{31}}{a_{21} a_{32}} \text {. }
$$

Let us see that $p, q, v_{1}, v_{3}$ and $t$ satisfy conditions (2.2), (2.3) and (2.4).

Let $A_{i j}$ denote the $2 \times 2$ submatrix of $A$ deleting row $i$ and column $j$ from $A$. Since $A$ is $(1,-1,-1)$-sign regular, we have

1. Since $\operatorname{det}\left(A_{33}\right)=\left(a_{11} a_{22}-a_{12} a_{21}\right)<0$, and $\operatorname{det}\left(A_{11}\right)<0$ we have $v_{1}<0$ and $v_{3}<0$. Then, condition (2.2) is fulfilled.

2. Since $\operatorname{det}\left(A_{31}\right)=\left(a_{12} a_{23}-a_{13} a_{22}\right)<0$ we deduce that $p>1$. Moreover $q>1$ since $\operatorname{det}\left(A_{13}\right)=\left(a_{21} a_{32}-a_{31} a_{22}\right)<0$, that is, $(2.3)$ holds.

3. Since $A$ and $T$ have the same determinant, then

$$
\operatorname{det}(T)=\frac{1-(p-1)(q-1)\left(v_{1}-1\right)\left(v_{3}-1\right)}{\left(v_{1}-1\right)\left(v_{3}-1\right)}
$$

is negative. This implies that $t<0$ which is the condition (2.4).

Therefore, $T$ has the required form and the entries satisfy the corresponding conditions $(2.2)-(2.4)$.

$(\Leftarrow)$ Conversely, suppose that $A$ is positively diagonally equivalent to matrix $T$ and the conditions (2.2), (2.3) and (2.4) are satisfied. In this case, it remains to prove that the matrix $T$ is $(1,-1,-1)$-sign regular since $A$ and $T$ have the same signature. Let us verify the sign of all determinants of order 1,2 and 3.

1. Determinants of order 1 . All of them are positive according to conditions (2.2) and (2.3).

2. Determinants of order 2. 
- $\operatorname{det}\left(T_{11}\right)=\left(\frac{v_{3}}{v_{3}-1}-1\right)=\frac{1}{v_{3}-1}<0$ and $\operatorname{det}\left(T_{33}\right)=\left(\frac{v_{1}}{v_{1}-1}-1\right)<$ 0 by $(2.2)$.

- $\operatorname{det}\left(T_{13}\right)=(1-q)<0$, and $\operatorname{det}\left(T_{31}\right)=(1-p)<0$ by $(2.3)$.

- $\operatorname{det}\left(T_{12}\right)=\left(\frac{v_{3}}{v_{3}-1}-q\right)<\frac{v_{3}}{v_{3}-1}-1=\frac{1}{v_{3}-1}<0$ by (2.3) and (2.2) . Similarly, $\operatorname{det}\left(T_{21}\right)=\left(\frac{v_{3}}{v_{3}-1}-p\right)<0, \operatorname{det}\left(T_{32}\right)=\left(\frac{v_{1}}{v_{1}-1}-p\right)<$ 0, and $\operatorname{det}\left(T_{23}\right)=\left(\frac{v_{1}}{v_{1}-1}-q\right)<0$.

- $\operatorname{det}\left(T_{22}\right)=\left(\frac{v_{1}}{v_{1}-1} \frac{v_{3}}{v_{3}-1}-p q\right)<0$ since $\left(\frac{v_{1}}{v_{1}-1}-p\right)<0$ and $\left(\frac{v_{3}}{v_{3}-1}-q\right)<0$.

3. Determinant of $T$. It is clear that $\operatorname{det}(T)<0$ by $(2.18),(2.17)$ and (2.15).

Therefore, $T$ is sign regular with signature $(1,-1,-1)$ and the given matrix $A$ also.

Theorem 2. A necessary and sufficient condition for three numbers, $u_{1}, u_{2}, u_{3}$ to be the diagonal entries of the combined matrix of a $(1,-1,-1)$-sign regular matrix is that $u_{i}>0, i=1,2,3$, and

$$
\sqrt{u_{1}}+\sqrt{u_{3}}-\sqrt{u_{2}}+1 \leq 0 .
$$

Proof. $\quad(\Rightarrow)$ Note that the diagonal entries of the combined matrix of a $(1,-1,-1)$-sign regular matrix $A$ are

$$
u_{i}=\frac{a_{i i} \operatorname{det} A_{i i}}{\operatorname{det} A}>0, \quad i=1,2,3 .
$$

In addition, any $(1,-1,-1)$-sign regular matrix is positively diagonally equivalent to the matrix $T$ of matrix- $T$ with the conditions (2.2)-(2.4) given in Lemma 2. As before, the combined matrix of $T, \Phi(T)$, has the following diagonal entries:

$$
u_{1}=\frac{v_{1}}{t}, u_{2}=\frac{v_{1} v_{3}-p q\left(v_{1}-1\right)\left(v_{3}-1\right)}{t}, u_{3}=\frac{v_{3}}{t}
$$

where

$$
t=1-(1-p)(1-q)\left(v_{1}-1\right)\left(v_{3}-1\right)<0 . \text { Then, }
$$

$$
p q=\frac{-u_{2} t+u_{1} u_{3} t^{2}}{\left(u_{1} t-1\right)\left(u_{3} t-1\right)}
$$


from the second diagonal entry. Moreover, we can write (2.4) as

$$
(1-p)(1-q)=\frac{1-t}{\left(-1+u_{1} t\right)\left(-1+u_{3} t\right)}
$$

From the last two equations, we have

$(1-q)\left(1-\frac{-u_{2} t+u_{1} u_{3} t^{2}}{q\left(-1+u_{1} t\right)\left(-1+u_{3} t\right)}\right)=\frac{1-t}{\left(-1+u_{1} t\right)\left(-1+u_{3} t\right)}$.

Simplifying, we have

$$
q^{2}\left(-1+u_{1} t\right)\left(-1+u_{3} t\right)+q\left(-1+u_{1}+u_{2}+u_{3}-2 u_{1} u_{3} t\right) t
$$

$$
-t\left(u_{2}-u_{1} u_{3} t\right)=0 .
$$

We have two real values $p, q>1$ satisfying equation (2.23). This implies that its discriminant is greater than or equal to zero, that is

$\left(t^{2} 4 u_{1} u_{3}+t\left(1-2 u_{1}+u_{1}^{2}-2 u_{2}-2 u_{1} u_{2}+u_{2}^{2}-2 u_{3}-2 u_{1} u_{3}-2 u_{2} u_{3}+u_{3}^{2}\right)+4 u_{2}\right) t \geq 0$. $(2.24)$

The corresponding equation, related to (2.24), has the solution $t=0$ plus the roots of the quadratic equation

$t^{2} 4 u_{1} u_{3}+t\left(1-2 u_{1}+u_{1}^{2}-2 u_{2}-2 u_{1} u_{2}+u_{2}^{2}-2 u_{3}-2 u_{1} u_{3}-2 u_{2} u_{3}+u_{3}^{2}\right)+4 u_{2}=0$.

To have all real roots, again its discriminant must be greater than or equal to zero, that is

$-64 u_{1} u_{2} u_{3}+\left(u_{1}^{2}+u_{2}^{2}+\left(-1+u_{3}\right)^{2}-2 u_{2}\left(1+u_{3}\right)-2 u_{1}\left(1+u_{2}+u_{3}\right)\right)^{2} \geq 0$.

The corresponding equation, related to (2.26), is of fourth degree with the variable $u_{2}$ and has the possible solutions:

$$
\begin{aligned}
& x_{1}=1+u_{1}+2 \sqrt{u_{1}} \sqrt{u_{3}}+u_{3}+2\left|\sqrt{u_{1}}-\sqrt{u_{3}}\right|, \\
& x_{2}=1+u_{1}-2 \sqrt{u_{1}} \sqrt{u_{3}}+u_{3}-2\left|\sqrt{u_{1}}-\sqrt{u_{3}}\right|, \\
& x_{3}=1+u_{1}-2 \sqrt{u_{1}} \sqrt{u_{3}}+u_{3}+2\left(\sqrt{u_{1}}+\sqrt{u_{3}}\right), \\
& x_{4}=1+u_{1}+2 \sqrt{u_{1}} \sqrt{u_{3}}+u_{3}+2\left(\sqrt{u_{1}}+\sqrt{u_{3}}\right) .
\end{aligned}
$$

Then, there are three possible regions for $u_{2}$ where $(2.26)$ holds. The first region is when $u_{2}$ is greater than or equal to the biggest root $x_{4}=$ 
$\left(1+\sqrt{u_{1}}+\sqrt{u_{3}}\right)^{2}$. In this case $u_{2} \geq x_{4}$ which is inequality (2.19) of our theorem.

The possibility that $u_{2}$ be in the other two regions is null, as we are going to see by contradiction.

The second possible region where the discriminant is not negative is determined by two of the three roots, $x_{1}, x_{2}, x_{3}$ depending on they are different and which one is the smallest. If we suppose without loss of generality that $u_{1} \geq u_{3}$, then $x_{1}=\left(1+\sqrt{u_{1}}-\sqrt{u_{3}}\right)^{2}, x_{2}=\left(1-\sqrt{u_{1}}+\sqrt{u_{3}}\right)^{2}$ and $x_{3}=\left(1-\sqrt{u_{1}}-\sqrt{u_{3}}\right)^{2}$. (Note that, if $u_{1} \leq u_{3}$, the roots $x_{1}$ and $x_{2}$ interchange its values.)

The expressions of the roots of (2.24) are

$$
\begin{aligned}
& t=\frac{1}{8 u_{1} u_{3}}\left(-1+2 u_{1}-u_{1}^{2}+2 u_{2}+2 u_{1} u_{2}-u_{2}^{2}+2 u_{3}+2 u_{1} u_{3}+2 u_{2} u_{3}-u_{3}^{2}\right. \\
& \left. \pm \sqrt{-64 u_{1} u_{2} u_{3}+\left(1-2 u_{1}+u_{1}^{2}-2 u_{2}-2 u_{1} u_{2}+u_{2}^{2}-2 u_{3}-2 u_{1} u_{3}-2 u_{2} u_{3}+u_{3}^{2}\right)^{2}}\right) .
\end{aligned}
$$

Since $u_{1}, u_{2}, u_{3}>0$, the roots of equation (2.27) are positive if and only if

$$
b=1-2 u_{1}+u_{1}^{2}-2 u_{2}-2 u_{1} u_{2}+u_{2}^{2}-2 u_{3}-2 u_{1} u_{3}-2 u_{2} u_{3}+u_{3}^{2}<0 .
$$

Note that the value of $b$ is negative when $u_{2}$ is between the two roots $b_{1}=1+u_{1}+u_{3}-2 \sqrt{u_{1}+u_{3}+u_{1} u_{3}}$ and $b_{2}=1+u_{1}+u_{3}+2 \sqrt{u_{1}+u_{3}+u_{1} u_{3}}$. Then, if $u_{2}$ goes into this region the function $b$ will be negative and the roots of $t$ positive which will be a contradiction with our point of depart where we have that $t<0$.

Let us see that our second region defined by $x_{1}, x_{2}$ or $x_{3}$ is included in the interval with bounds $b_{1}$ and $b_{2}$.

First note that $b_{2}>x_{1}, x_{2}, x_{3}$ since

$$
\begin{aligned}
& b_{2}-x_{1}=2\left(\sqrt{u_{3}}-\sqrt{u_{1}}+\sqrt{u_{1} u_{3}}+\sqrt{u_{1}+u_{3}+u_{1} u_{3}}\right) \\
& b_{2}-x_{2}=2\left(\sqrt{u_{1}}-\sqrt{u_{3}}+\sqrt{u_{1} u_{3}}+\sqrt{u_{1}+u_{3}+u_{1} u_{3}}\right) \\
& b_{2}-x_{3}=2\left(\sqrt{u_{1}}+\sqrt{u_{3}}-\sqrt{u_{1} u_{3}}+\sqrt{u_{1}+u_{3}+u_{1} u_{3}}\right)
\end{aligned}
$$

where the three differences are positive because

$$
\sqrt{u_{1}+u_{3}+u_{1} u_{3}}>\sqrt{u_{1}}, \sqrt{u_{3}}, \sqrt{u_{1} u_{3}} .
$$

For the lower bound $b_{1}$ we can see that $b_{1}$ is less than at least two of the three roots $x_{1}, x_{2}, x_{3}$, two of them defining the second region. 
- For all $u_{1} \geq u_{3}>0$, we have

$$
b_{1}<x_{1} \Leftrightarrow \sqrt{u_{3}}+\sqrt{u_{1} u_{3}}<\sqrt{u_{1}}+\sqrt{u_{1}+u_{3}+u_{1} u_{3}}
$$

and the second inequality holds since $\sqrt{u_{1}} \geq \sqrt{u_{3}}$ and $\sqrt{u_{1}+u_{3}+u_{1} u_{3}}>\sqrt{u_{1} u_{3}}$.

- If $u_{1} \geq 1$, then

$$
b_{1}<x_{3} \Leftrightarrow \sqrt{u_{1}}+\sqrt{u_{3}}<\sqrt{u_{1} u_{3}}+\sqrt{u_{1}+u_{3}+u_{1} u_{3}}
$$

and the second inequality holds because $\sqrt{u_{1} u_{3}} \geq \sqrt{u_{3}}$ and $\sqrt{u_{1}+u_{3}+u_{1} u_{3}}>\sqrt{u_{1}}$.

- If $u_{1}<1$, we have

$$
b_{1}<x_{2} \Leftrightarrow \sqrt{u_{1}}+\sqrt{u_{1} u_{3}}<\sqrt{u_{3}}+\sqrt{u_{1}+u_{3}+u_{1} u_{3}}
$$

and the second inequality holds since $\sqrt{u_{3}}>\sqrt{u_{1} u_{3}}$ and $\sqrt{u_{1}+u_{3}+u_{1} u_{3}}>\sqrt{u_{1}}$.

Then, the second region is included in $] b_{1}, b_{2}\left[\right.$ and $u_{2}$ cannot be in this interval because $t$ would be positive.

Let us study the third region, of values of $u_{2}$, where the discriminant (2.26) is not negative. This case corresponds when $u_{2}$ is less than or equal to the smallest root, that is, $u_{2} \leq x_{1}, x_{2}, x_{3}$. Again, without loss of generality, we suppose that $u_{1} \geq u_{3}$.

- $u_{3} \leq 1$, then

$$
\begin{aligned}
u_{2}-x_{3} & =u_{2}-\left(1-\sqrt{u_{1}}-\sqrt{u_{3}}\right)^{2} \\
& =\left(u_{2}-1-u_{1}-u_{3}\right)+2\left(\sqrt{u_{1}}+\sqrt{u_{3}}-\sqrt{u_{1} u_{3}}\right) \\
& =F+2 G>0
\end{aligned}
$$

since $F$ and $G$ are positive. This is because from the values of $u_{1}, u_{2}, u_{3}$ of the equation (2.20) we obtain

$$
F=\frac{\left(v_{1}-1\right)\left(v_{3}-1\right)(2-p-q)-2}{t}>0 .
$$

Moreover, in this case, $u_{1} u_{3} \leq u_{1}$ and then $G>0$. 
- $u_{3}>1$, then

$$
\begin{aligned}
u_{2}-x_{2} & =u_{2}-\left(1-\sqrt{u_{1}}+\sqrt{u_{3}}\right)^{2} \\
& =\left(u_{2}-1-u_{1}-u_{3}\right)+2\left(\sqrt{u_{1}}-\sqrt{u_{3}}+\sqrt{u_{1} u_{3}}\right)>0
\end{aligned}
$$

which is positive because $u_{1} \geq u_{3}$ and so $\sqrt{u_{1}} \geq \sqrt{u_{3}}$ and the considerations of the above term $F$.

Then, $u_{2}$ values cannot be in the third region.

Therefore, the values of $u_{2}$ for which the discriminant (2.26) is not negative, are only in the first region. Hence, we can conclude that, if $u_{1}, u_{2}, u_{3}$ are the diagonal entries of a $(1,-1,-1)$-sign regular matrix then, $u_{1}>0, u_{2}>0, u_{3}>0$ and the equation (2.19) holds.

Conversely, let $u_{1}, u_{2}, u_{3}$ be three positive numbers satisfying the equation (2.19). We are looking for $v_{1}, v_{3}<0, p, q>1$ and $t<0$ so that the equation

$$
u_{1}=\frac{v_{1}}{t}, u_{2}=\frac{v_{1} v_{3}-p q\left(v_{1}-1\right)\left(v_{3}-1\right)}{t}, u_{3}=\frac{v_{3}}{t}
$$

and $t=1-(1-p)(1-q)\left(v_{1}-1\right)\left(v_{3}-1\right)$ hold. Then, we will built a $(1,-$ 1,-1)-sign regular matrix $T$ as in Lemma 2 with the structure of matrix- $T$ and conditions (2.15)-(2.17) such that $u_{1}, u_{2}, u_{3}$ are the diagonal entries of its combined matrix $\Phi(T)$.

We are going to argue in an opposite way as in the last part of the direct proof. From condition (2.19), the inequality (2.26) holds and then equation (2.25) has real the two nonzero roots described in (2.27).

Since $u_{1}>0, u_{2}>0, u_{3}>0$, the last two roots are negative provided that

$$
b=1-2 u_{1}+u_{1}^{2}-2 u_{2}-2 u_{1} u_{2}+u_{2}^{2}-2 u_{3}-2 u_{1} u_{3}-2 u_{2} u_{3}+u_{3}^{2}>0 .
$$

This last inequality holds from the inequality (2.19) of our theorem. That is, we can write

$$
u_{2}=\left(1+\sqrt{u_{1}}+\sqrt{u_{3}}\right)^{2}+\epsilon, \text { with } \epsilon \geq 0
$$

and then

$b=\epsilon^{2}+4 \epsilon \sqrt{u_{1}}+4 \epsilon \sqrt{u_{3}}+8 \sqrt{u_{1}} \sqrt{u_{3}}+4 \epsilon \sqrt{u_{1}} \sqrt{u_{3}}+8 u_{1} \sqrt{u_{3}}+8 u_{3} \sqrt{u_{1}}>0$

for all $\epsilon \geq 0$. 
Then, if we choose a negative value of $t$ between these negative roots, we obtain $v_{1}=u_{1} t<0, v_{3}=u_{3} t<0$ and $p, q>0$ where $p$ and $q$ are the solutions of equation (2.23).

It remains to prove that $p, q>1$. Our condition (2.19) implies $u_{2} \geq$ $u_{1}+u_{3}+2 \sqrt{u_{1}} \sqrt{u_{3}}+2 \sqrt{u_{1}}+2 \sqrt{u_{3}}+1$. Then

$$
u_{2}+1>u_{1}+u_{3},
$$

that is

$$
-u_{2} t-t>-u_{1} t-u_{3} t
$$

since $t<0$. Adding $1+u_{1} u_{3} t^{2}$ to both terms, we have

$$
-u_{2} t-t+u_{1} u_{3} t^{2}+1>\left(u_{1} t-1\right)\left(u_{3} t-1\right)
$$

and so

$$
\sqrt{-u_{2} t-t+u_{1} u_{3} t^{2}+1}>\sqrt{\left(u_{1} t-1\right)\left(u_{3} t-1\right)} .
$$

The first square root satisfies

$$
\sqrt{-u_{2} t+u_{1} u_{3} t^{2}}+\sqrt{1-t}>\sqrt{-u_{2} t-t+u_{1} u_{3} t^{2}+1}
$$

and then

$$
\sqrt{\frac{-u_{2} t+u_{1} u_{3} t^{2}}{\left(u_{1} t-1\right)\left(u_{3} t-1\right)}}+\sqrt{\frac{1-t}{\left(u_{1} t-1\right)\left(u_{3} t-1\right)}}>1 .
$$

Then, from Remark 1 the solutions of equations (2.21) and (2.22) are not in $(0,1)$. Since $p, q>0$ and $(1-p)(1-q)>0$ by equation $(2.22)$, then $p, q>1$.

Then, we have found a solution for the unknowns $t, v_{1}, v_{3}, p$ and $q$ satisfying conditions (2.2)-(2.4) in which case the matrix $T$ is $(1,-1,-1)$-sign regular by Lemma 2 . Note that the diagonal elements of its combined matrix are

$$
\operatorname{diag}(\Phi(T))=\left(\frac{v_{1}}{t}, \frac{v_{1} v_{3}-p q\left(v_{1}-1\right)\left(v_{3}-1\right)}{t}, \frac{v_{3}}{t}\right),
$$

which are precisely the elements $\left(u_{1}, u_{2}, u_{3}\right)$ of the equation (2.28).

Example 2. $(\Rightarrow)$ Consider the (1,-1,-1)-sign regular matrix

$$
T=\left[\begin{array}{rrr}
0.5 & 1 & 100 \\
1 & 1 & 1 \\
5 & 1 & 0.25
\end{array}\right]
$$


Note that $p, q>1, v_{1}=-1<0, v_{3}=-1 / 3<0$ and $t=-1055<0$.

The diagonal entries of its combined matrix are $u_{1}=1 / 1055, u_{2}=$ $1333 / 1055, u_{3}=1 / 3165$ fulfilling

$$
\left(\sqrt{u_{1}}+\sqrt{u_{3}}+1\right)^{2} \approx 1,09948<u_{2} \approx 1.26351 .
$$

$(\Leftarrow)$ Consider $u_{1}=u_{3}=1$. Since $\left(\sqrt{u_{1}}+\sqrt{u_{3}}+1\right)^{2}=9$, we take $u_{2}=10$. To obtain the $(1,-1,-1)$-sign regular matrix $T$, we need to compute the negative roots expressed in (2.27): $t_{1}=-8$ and $t_{2}=-5 / 4$. Then we take $t=-2$. With these value of $t<0$ we can compute $v_{1}=u_{1} / t=v_{3}=$ $u_{3} / t=-1 / 2$ and obtain the roots of (2.23), the values (greater than 1 ) of $p$ and $q$ are

$$
p=\frac{4}{3}, q=2 \quad \text { or } \quad p=2, q=\frac{4}{3} .
$$

Then, $\{1,10,1\}$ are the diagonal entries of the combined matrix of $T$, where $T$ is the $(1,-1,-1)$-sign regular matrix built as in Lemma 2. That is, $T$ can be any of the following matrices

$$
T_{1}=\left[\begin{array}{ccc}
\frac{2}{3} & 1 & 2 \\
1 & 1 & 1 \\
\frac{4}{3} & 1 & \frac{2}{3}
\end{array}\right], \quad T_{2}=\left[\begin{array}{ccc}
\frac{2}{3} & 1 & \frac{4}{3} \\
1 & 1 & 1 \\
2 & 1 & \frac{2}{3}
\end{array}\right]
$$

(Symmetric case) Taking $u_{1}=4, u_{3}=9$ and $u_{2}=\left(\sqrt{u_{1}}+\sqrt{u_{3}}+1\right)^{2}=$ 36 , we obtain only one negative root for $t: t=-1$. With these value of $t<0$, we can obtain $p=q=6 / 5>1, v_{1}=-4<0$ and $v_{3}=-9$.

Then, the (1,-1,-1)-sign regular matrix is, for instance,

$$
T_{1}=\left[\begin{array}{ccc}
\frac{4}{5} & 1 & \frac{6}{5} \\
1 & 1 & 1 \\
\frac{6}{5} & 1 & \frac{9}{10}
\end{array}\right] \quad \text { or } T_{2}=\left[\begin{array}{rrr}
8 & 10 & 12 \\
10 & 10 & 10 \\
12 & 10 & 9
\end{array}\right]
$$

\section{Acknowledgements}

Supported by Spanish Ministerio de Economía y Competitividad grants MTM2017- 85669-P and MTM2017-90682-REDT and Dominican Republic FONDOCYT grant number 2015-1D2-166. 


\section{References}

[1] P. Alonso and M. L. Serrano, "Combined matrices of almost strictly sign regular matrices", Journal of computational and applied mathematics, vol. 354, pp. 144-151, Jul. 2019, doi: 10.1016/ j.cam.2018.09.029

[2] E. Bristol, "On a new measure of interaction for multivariable process control", IEEE transactions on automatic control, vol. 11, no. 1, pp. 133-134, Jan. 1966, doi: 10.1109/ TAC.1966.1098266

[3] R. Bru, M. Gassó, I. Giménez, and M. Santana, "Combined matrices of sign regular matrices", Linear algebra applications, vol. 498, pp. 88-98, Jun. 2016. doi: 10.1016/ j.laa.2014.12.010

[4] R. Bru, M. Gassó, I. Giménez, and M. Santana, "Diagonal entries of the combined matrix of a totally negative matrix", Linear and multilinear algebra, vol. 65, no. 10, pp 1971-1984, 2017, doi: $10.1080 / 03081087.2016 .1261079$

[5] M. Fiedler, "Relations between the diagonal entries of an M-matrix and of its inverse", Matematicko-fyzikálny časopis, vol. 12, no. 2, pp. 123-128, 1962. [On line]. Available: https:// bit.ly/2XHudbq

[6] M. Fiedler, "Relations between the diagonal entries of two mutually inverse positive definite matrices", Czechoslovak mathematics journal, vol. 14, no.1, pp. 39-51, 1964, doi: 10.21136/ cmj.1964.100600

[7] M. Fiedler and T. L. Markham, "Combined matrices in special classes of matrices", Linear algebra applications, vol. 435, no. 8, pp. 1945-1955, Oct. 2011, doi: 10.1016/ j.laa.2011.03.054

[8] R. A. Horn and C. R. Johnson, Topics in matrix analysis. Cambridge: Cambridge University Press, 1991, doi: 10.1017/ CBO9780511840371

[9] J. M. Peña, "On nonsingular sign regular matrices", Linear algebra and applications, vol. 359, no. 1-3, pp. 91-100, Jan. 2003, doi: $10.1016 /$ S0024-3795(02)00437-8 